\title{
THE UNIVERSITY of EDINBURGH
}

\author{
Edinburgh Research Explorer
}

\section{External coding and salience in the tactile Simon effect}

\section{Citation for published version:}

Medina, J, Theodoropoulos, N, Liu, Y, Reyes, P \& Gherri, E 2019, 'External coding and salience in the tactile Simon effect', Acta Psychologica, vol. 198, 102874. https://doi.org/10.1016/j.actpsy.2019.102874

\section{Digital Object Identifier (DOI):}

10.1016/j.actpsy.2019.102874

\section{Link:}

Link to publication record in Edinburgh Research Explorer

\section{Document Version:}

Peer reviewed version

\section{Published In:}

Acta Psychologica

\section{General rights}

Copyright for the publications made accessible via the Edinburgh Research Explorer is retained by the author(s) and / or other copyright owners and it is a condition of accessing these publications that users recognise and abide by the legal requirements associated with these rights.

\section{Take down policy}

The University of Edinburgh has made every reasonable effort to ensure that Edinburgh Research Explorer content complies with UK legislation. If you believe that the public display of this file breaches copyright please contact openaccess@ed.ac.uk providing details, and we will remove access to the work immediately and investigate your claim. 
External coding and salience in the tactile Simon effect

Jared Medina, Ph.D. ${ }^{1}$, Nikolaos Theodoropoulos ${ }^{2}$, Yuqi Liu ${ }^{1}$, Patrick G. Reyes ${ }^{1} \&$ Elena Gherri, Ph.D. ${ }^{2}$

Department of Psychology, University of Delaware ${ }^{1}$

Department of Psychology, The University of Edinburgh ${ }^{2}$

\section{Corresponding address:}

Jared Medina

University of Delaware

105 The Green, Room 108

Newark, DE 19716

Phone: 302-831-8724

Fax: 302-831-3645

E-mail: jmedina@psych.udel.edu

Words in manuscript body: 10805 


\begin{abstract}
Previous studies have demonstrated a tactile Simon effect in which stimulus codes are generated based on the stimulated hand, not on limb position in external space (the somatotopic Simon effect). However, given evidence from visual Simon effect studies demonstrating that multiple stimulus codes can be generated for a single stimulus, we examined whether multiple stimulus codes can be generated for tactile stimuli as well. In our first experiment using four stimulators (two on each side of the hand), we found novel evidence for a hand-centered Simon effect, along with the typical somatotopic Simon effect. Next, we examined whether the potential salience of these somatotopic codes could be reduced, by testing only one hand with two stimulators attached. In Experiments 2-4, we found a strong handcentered Simon effect with a diminished somatotopic Simon effect, providing evidence that stimulus salience can change the weighting of somatosensory stimulus coding. Finally, we also found novel evidence that the hand-centered Simon effect is coded in external, not somatotopic, coordinates. Furthermore, the diminished somatotopic Simon effect when testing on one hand only provides evidence that salience is an important factor in modulating the tactile Simon effect.
\end{abstract}

Keywords: Simon effect, tactile, frames of reference, salience 


\section{Introduction}

When a touch is presented to the skin surface, its location can be represented based on a variety of different reference frames. Most examinations of tactile spatial representations have focused on two general categories of reference frames. First are somatotopic reference frames, in which the represented location is anchored to the skin surface and does not change when body position changes. Second are external reference frames, in which the represented location of touch is based on its position in external space. For example, imagine a tactile stimulus presented to the tip of the left index finger with the arms uncrossed and crossed. In a somatotopic frame of reference, that tactile stimulus is represented in the same location either with the hands crossed or uncrossed. Whereas in a (for example) trunk-centered external frame of reference, that stimulus is represented on the left side of the body with the arms uncrossed, and the right side with the arms crossed.

One experimental paradigm that has been used to examine spatial representations is the Simon task (Simon \& Small, 1969). In the Simon effect, participants respond faster when both the stimulus and response are on the same side of space versus different sides of space in a non-spatial task. This has been observed in vision (Wallace, 1971), touch (Hasbroucq \& Guiard, 1992) and audition (Simon \& Rudell, 1967; Simon \& Small, 1969). When the stimulus and response are on different sides, a task-irrelevant code for stimulus location interferes with a task-relevant response code. This interference between the task-irrelevant and task-relevant spatial codes causes a slower response. When the stimulus and response are on the same side, the task-relevant and task-irrelevant spatial codes are consistent, and responses are faster because there is less spatial code competition or interference.

Nearly all explanations of the Simon effect propose that a spatial code is generated for stimuli and responses. An important question is how left and right are defined in these spatial codes. In the visual (Riggio, Gawryszewski, \& Umilta, 1986; Wallace, 1971) and auditory (Roswarski \& Proctor, 2000; Simon, Hinrichs, \& Craft, 1970) modalities, stimuli are encoded in an external frame of reference. Medina, McCloskey, Coslett \& Rapp (2014) examined the reference frame for spatial code generation in the tactile Simon effect. In these experiments, participants had one tactile stimulator placed on each hand, and were presented with a high- or low-intensity tactile stimulus. Participants were told to lift a foot pedal based on the instructed stimulus-response mapping (e.g. lift the left foot pedal for high intensity stimuli, right for low intensity stimuli). Participants were tested with their hands crossed and uncrossed, to examine if the tactile Simon effect operated based on a somatotopic or external frame of reference. There 
was strong evidence for a somatotopic Simon effect, such that participants were faster when the left hand was stimulated with a left foot response, regardless of whether the hands were crossed or uncrossed.

Our experiments in the current manuscript will follow up on previous results with the tactile Simon effect focusing on two topics. Previous research has only demonstrated a tactile Simon effect based on a somatotopic frame of reference. However, it is possible that the tactile Simon effect can be generated based on multiple frames of reference, including ones that are not somatotopic. Experiments with the visual Simon effect have provided evidence that visual stimulus codes can be generated by multiple representations with external frames of reference. For example, Lamberts, Tavernier \& d'Ydewalle (1992) presented subjects with a Simon effect task in which stimuli were presented in one of eight potential positions, four in each hemispace. A fixation point emerged in the center of either hemispace (creating separate hemifields), with two boxes then presented either to the left or right of the displayed fixation point. The response stimulus was presented within one of these boxes, and participants responded with either the left or right hand depending on the target presented. In this experiment, each stimulus was in a different hemifield, hemispace, and relative position (i.e. left or right in the two target squares). They found a significant visual Simon effect for all three reference frames, concluding that stimuli generate multiple spatial codes based on different representations with different reference frames (see also Roswarski \& Proctor, 1996 for similar results).

In our previous published research with the tactile Simon effect, we only presented one stimulus to each hand. Although this manipulation distinguishes between somatotopic and external reference frames, a second possibility is that stimulus locations are also encoded based on additional frames of reference. An external reference frame is a general category that encompasses a number of different potential representations, each with their own frame of reference. For example, assume that a participant is positioned with their hands on a table, palms down, each in their typical hemispace (uncrossed), and is stimulated on the right side of their left thumb. That stimulus would be encoded as left in a reference frame in which the midline is generated from the center of the participant's trunk (a trunk-centered external frame of reference). However, that stimulus location can also be represented in a hand-centered frame of reference, in which the midline is the center of the long axis of the hand, or even in a fingercentered frame of reference, with the midline being the long axis of the finger. Studies of individuals with tactile extinction, a deficit in which participants fail to respond to the contralesional stimulus when simultaneously presented with two bilateral stimuli, have provided evidence for multiple frames of 
reference being involved in tactile processing. Moscovitch \& Behrmann (1994) report individuals who extinguished stimuli presented on the contralesional side of the wrist, regardless of whether the hand was positioned palm-up or palm-down. These results are consistent with a deficit based on the relative position of the two tactile stimuli, or a deficit in a "hand-centered" reference frame (see also Tinazzi, Ferrari, Zampini, \& Aglioti, 2000).

There is evidence from individuals with tactile extinction for multiple reference frames involved in representing tactile stimuli (Moscovitch \& Behrmann, 1994; Tinazzi, Ferrari, Zampini \& Aglioti, 2000). Furthermore, evidence from the visual Simon effect also demonstrates that multiple spatial codes can be generated, each with their own frame of reference (Lamberts, Tavernier \& d'Ydewalle, 1992; Roswarski \& Proctor, 1996). However, it is unknown whether tactile stimulus codes are generated from representations with non-somatotopic frames of reference. Therefore, our first question will examine whether multiple frames of reference are involved in the tactile Simon effect.

Second, we examined whether somatotopic stimulus codes are always generated, regardless of task demands. Although the Simon effect is a typically robust finding, recent studies have shown that it can be diminished, or disappear, under particular conditions. Ruzzoli \& Soto-Faraco (2017) presented participants with a mixed-modality Simon effect task, in which the modality of the stimulus varied on each trial, either visual and tactile (Experiments 1-4) or visual and auditory (Experiment 5). In the mixed visual-auditory task, they found a robust Simon effect in both modalities. However, in the mixed visualtactile task, they found that only the tactile Simon effect remained, with no evidence for a visual Simon effect across multiple experiments. These results provide evidence that spatial codes generated in the Simon effect are not obligatorily generated, and can vary based on presented modalities (see also Castro, Soto- Faraco, Morís Fernández, \& Ruzzoli, 2018). In some of our experiments, participants wore multiple tactile stimulators on a single hand, allowing us to examine whether the Simon effect is generated in a hand-centered frame of reference. In this condition, tactile stimuli could also be coded based on a somatotopic frame of reference (i.e. the hand that was stimulated). However, as only one hand was tested, it is possible that the somatotopic frame of reference was not salient due to a lack of behavioural relevance, resulting in a diminished somatotopic Simon effect. Therefore, we examined whether presenting tactile stimuli to one hand only modulated the strength of the somatotopic Simon effect. 


\section{Experiment 1: Is there a hand-centered tactile Simon effect?}

In Experiment 1, we ran a standard tactile Simon task paradigm in which participants were asked to make a foot pedal response to tactile stimuli presented at different intensities (e.g. press the left foot for a high intensity stimulus; right foot for a low intensity stimulus). Previous tactile Simon task studies have had one stimulator on each hand, whereas in our study participants had two tactile stimulators attached to each hand (one on the thumb, one on the pinky). Each stimulus could be encoded, both in a somatotopic frame of reference (left hand, right hand) and in an external, hand-centered frame of reference (left and right side of the hand). Given that multiple stimulus codes have been generated in visual Simon effect experiments (Lamberts et al., 1992; Roswarski \& Proctor, 1996), along with evidence

suggesting hand-centered coding in tactile extinction (Moscovitch \& Behrmann, 1994), we hypothesized that tactile stimuli would be encoded both based on the stimulated hand ("somatotopic" encoding) and based on the side of the stimulus in an external hand-centered frame of reference. Therefore, we predicted a significant somatotopic and hand-centered Simon effect. However, given the tight coupling between somatosensory and motor systems, it is possible that hand-centered spatial codes are not coded, or are not strong enough to generate a Simon effect. The alternative prediction was a somatotopic, but no external hand-centered, Simon effect.

\subsection{Methods}

All research for Experiment 1 was conducted at the University of Delaware with approval from the local Institutional Review Board. Furthermore, all research protocols were preregistered on Open Science Framework. Preregistered research protocols, scripts, and all data can be found at https://osf.io/dw52r/.

\section{Participants}

The Simon effect is typically a quite robust effect. However, given that we examined whether there is evidence for a tactile Simon effect in multiple frames of reference, and since we assume that a hand-centered Simon effect is likely smaller than a somatotopic effect, we designed an experiment to detect a moderate to large effect size $(\mathrm{dz}=.6)$ with $90 \%$ power, and an alpha of .05 (two-tailed). Using G*Power 3.1.9.2. for t-tests (Means: difference between two dependent means (matched pairs)), the total recommended sample size was 32 . 
Forty-three participants were tested, with six excluded due to experiment issues: one for incorrect registration of foot pedals, one for ending the experiment early, one because the grey noise stopped playing during a block and three due to foot pedal malfunctions during part of the block. After those exclusions, we analysed the first 32 participants that met inclusion criteria, as stated in our preregistration plan (mean age: 18.6, 18-22, 20 females, three left-handed). All participants were undergraduates at the University of Delaware, who were reimbursed via participation credits for an Introduction to Psychology course.

\subsubsection{Apparatus, stimuli and procedure}

Participants were seated at a table with a flat-screen monitor facing them, with their trunk midline aligned with the center of the screen. The participant had four tactile stimulators (Tactaid VBW32 Skin Transducers, Audiological Engineering Corporation, Somverville, MA) strapped onto the dorsal surface of their left pinky finger, left thumb, right pinky finger, and right thumb, with their hands positioned palms down. These tactile stimulators were attached to a Dell Precision T1650 PC running Psych Toolbox. This PC was connected to an external multi-channel sound card (Gigaport HD+), which was connected to two PYLE PTA2 40-watt stereo power amplifiers. The volume for the PC was set to 100, with the volume for each amplifier set at 50. To mask any potential noise from the tactile stimulators, participants listened to grey noise via headphones.

Two foot pedals were located underneath the table, $30 \mathrm{~cm}$ to the left or right of their trunk midline. These foot pedals were attached to an E-Prime button box for data collection. Participants responded by pressing a foot pedal. Tactile stimuli were made as audio files in Audacity 2.1.3. Stimuli were 250-ms high-intensity $(250-\mathrm{Hz}$ pulse set at $-10 \mathrm{~dB})$ or low-intensity $(250-\mathrm{Hz}$ pulse set at $-25 \mathrm{~dB})$ tactile stimuli. Both stimuli were suprathreshold, and easily discriminable in pilot testing.

\subsubsection{Pre-screener}

To ensure that participants could differentiate between high- and low-intensity stimuli, we presented them with a brief screener before the experiment. To start, participants were given examples of high and low intensity stimuli to each of the four tactile stimulators. Next, 20 screening trials were presented in which participants were instructed to respond by verbally indicating whether the stimulus was high or low intensity, with no feedback. Participants had two opportunities to get 19/20 trials correct to move onto the main experiment. All participants successfully passed the pre-screener.

\subsubsection{Experimental Procedure}


Before each trial, participants were presented with a fixation point on the center of the screen. Next, 800-1200 ms after fixation onset, participants were presented with a high- or low-intensity tactile stimulus to one of the four tactile stimulators. Participants were instructed to respond as quickly as possible (by pressing a foot pedal based on the instructed stimulus-response mapping) as to whether the stimulus was high- or low-intensity. After responding, participants received feedback on the screen as to whether they were correct or incorrect for $500 \mathrm{~ms}$, followed by the next trial. If no response was recorded $1500 \mathrm{~ms}$ after stimulus onset, the trial was ended with a message of "no response" showing as feedback.

Each participant was presented with four blocks, in which hand position (crossed or uncrossed) was modified in an ABBA order. Each block consisted of 16 practice trials followed by 96 experimental trials, counter-balanced for stimulus location and intensity. Stimulus-response mapping (A: left foot pedal, high-intensity tactile stimulus; right foot pedal, low-intensity tactile stimulus; B: left foot pedal, low-intensity tactile stimulus; right foot pedal, high-intensity tactile stimulus) was modified across subjects (i.e. half had mapping A, half had mapping B).

\subsection{Results}

We excluded $0.95 \%$ of trials that were outliers as defined in our preregistration document (RT values $>3 S D$ s above or below the participant's mean RT for the block), along with anticipatory responses before the stimulus was presented, or trials where the subject failed to respond. Trunk-Centered Somatotopic Congruency refers to congruency based on the anatomically defined side of the body, such that any stimulus presented to the left hand would be coded as "left" regardless of its position in space, or regardless of whether other hands are tested. Hand-Centered Congruency refers to congruency based on the side of the hand that was stimulated. For example, a stimulus on the left pinky (palm down) is on the left side of the hand, whereas a stimulus on the left thumb (palm down) is on the right side of the hand. A left-foot response to the left pinky stimulus would be congruent in a hand-centered frame of reference; a right-foot response to that same stimulus would be incongruent in a hand-centered frame of reference.

\subsubsection{Reaction Times}

Reaction times were log-transformed and analyzed in a 2 (Hand Position) x 2 (Trunk-Centered Somatotopic Congruency) x 2 (Hand-Centered Congruency) analysis of variance (ANOVA). First, there was a main effect of hand position, as participants were $15 \mathrm{~ms}$ faster on crossed-hand trials (619 ms) versus uncrossed-hand trials $(634 \mathrm{~ms}), F(1,31)=7.06, p=.012, \eta_{p}^{2}=0.19$, consistent with previous 
work (Medina et al., 2014). There was also a main effect of trunk-centered somatotopic congruency, as participants were $28 \mathrm{~ms}$ faster for trunk-centered somatotopically congruent trials $(613 \mathrm{~ms})$ than for trunk-centered somatotopically incongruent trials $(641 \mathrm{~ms}), F(1,31)=77.75, p<.001, \eta_{p}^{2}=0.71$. A significant hand-centered congruency effect was also observed, as participants were $13 \mathrm{~ms}$ faster for hand-centered congruent trials (620 ms) compared to hand-centered incongruent trials $(633 \mathrm{~ms}), F(1,31)$ $=23.64, p<.001, \eta_{p}^{2}=0.43$. Finally, there was a significant trunk-centered somatotopic congruence by hand position interaction, $F(1,31)=36.68, p<.001, \eta_{p}^{2}=0.54$, as the Simon effect was larger for uncrossed trials $(45 \mathrm{~ms})$ than for crossed trials $(10 \mathrm{~ms})$. In both postures, there was a significant Simon effect (uncrossed, $\mathrm{t}(31)=10.6, \mathrm{p}<.001 ;$ crossed, $\mathrm{t}(31)=2.46, \mathrm{p}=.019$ ).

Based on our preregistration document, we also analysed log-transformed reaction times in a

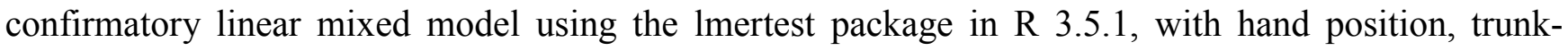
centered somatotopic congruency, and hand-centered congruency as fixed effects. The following random slopes and intercepts were also included: (1+hand position|subject), (1+trunk-centered somatotopic congruency|subject), (1+hand-centered congruency|subject). As in the ANOVA, there were significant main effects of hand position $(b=-.025, t=-2.65, p=.013)$, trunk-centered somatotopic congruency $(b$ $=-.048, \mathrm{t}=8.82, \mathrm{p}<.001)$, and hand-centered congruency $(\mathrm{b}=-.018, \mathrm{t}=-4.66, \mathrm{p}<.001)$; along with $\mathrm{a}$ significant trunk-centered somatotopic congruency by hand position interaction $(b=.056, t=6.01, p<$ .001). No other comparisons were significant.

\subsubsection{Accuracy}

Errors were analyzed in a binomial generalized linear mixed model (GLMM) using the lmertest package in $\mathrm{R}$ version 3.5.1, with the same fixed effects and random effects structure as in the reaction time linear mixed model in 2.2.1.

First, there was a significant main effect of trunk-centered somatotopic congruency $(b=.674, z$ $=5.82, p<.001)$, with fewer errors on trunk-centered somatotopically congruent trials $(3.5 \%)$ than in trunk-centered somatotopically incongruent trials $(6.3 \%)$. There was also a main effect of hand-centered congruence $(\mathrm{b}=.377, z=3.00, p=.003)$, with fewer errors on hand-centered congruent trials $(4.2 \%)$ than hand-centered incongruent trials $(5.6 \%)$. There was also a significant interaction between hand position and trunk-centered somatotopic congruency $(\mathrm{b}=-.766, z=-2.70, p=.007)$, with a larger Simon effect with the hands uncrossed (4.4\%) versus crossed (1.3\%). 
Accuracy was also analyzed in a 2 (Hand Position) $x 2$ (Trunk-Centered Somatotopic Congruency) x 2 (Hand-Centered Congruency) analysis of variance (ANOVA). The ANOVA reported the same significant effects as the GLMM: main effects of trunk-centered somatotopic congruency, $F(1$, $31)=33.957, p<.001, \eta_{p}^{2}=0.52$; hand-centered congruency, $F(1,31)=7.39, p=.011, \eta_{p}^{2}=0.19$; and an interaction between hand position and trunk-centered somatotopic congruency, $F(1,31)=8.47, p<$ $.001, \eta_{p}^{2}=0.21$. Additionally, there was also a significant hand position by hand-centered congruency interaction, $F(1,31)=4.88, p=.035, \eta_{p}^{2}=0.14$, that did not reach significance in the mixed model $(z=$ $1.864, p=.062)$. In the interaction, the hand-centered Simon effect was larger with the hands crossed $(2.4 \%)$ versus uncrossed $(.2 \%)$.

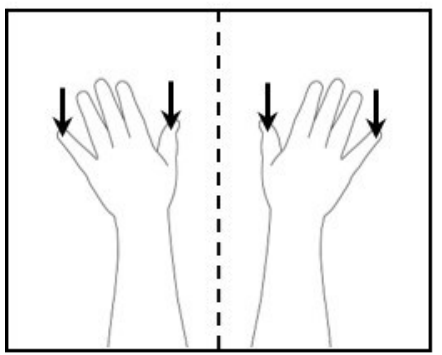

Uncrossed hands

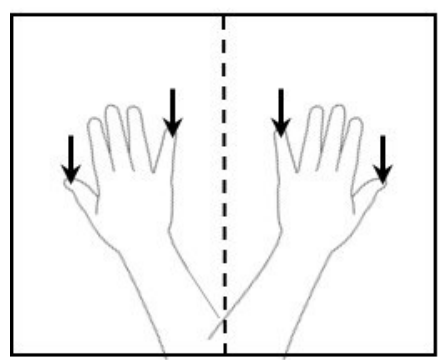

Crossed hands

Congruent

Incongruent

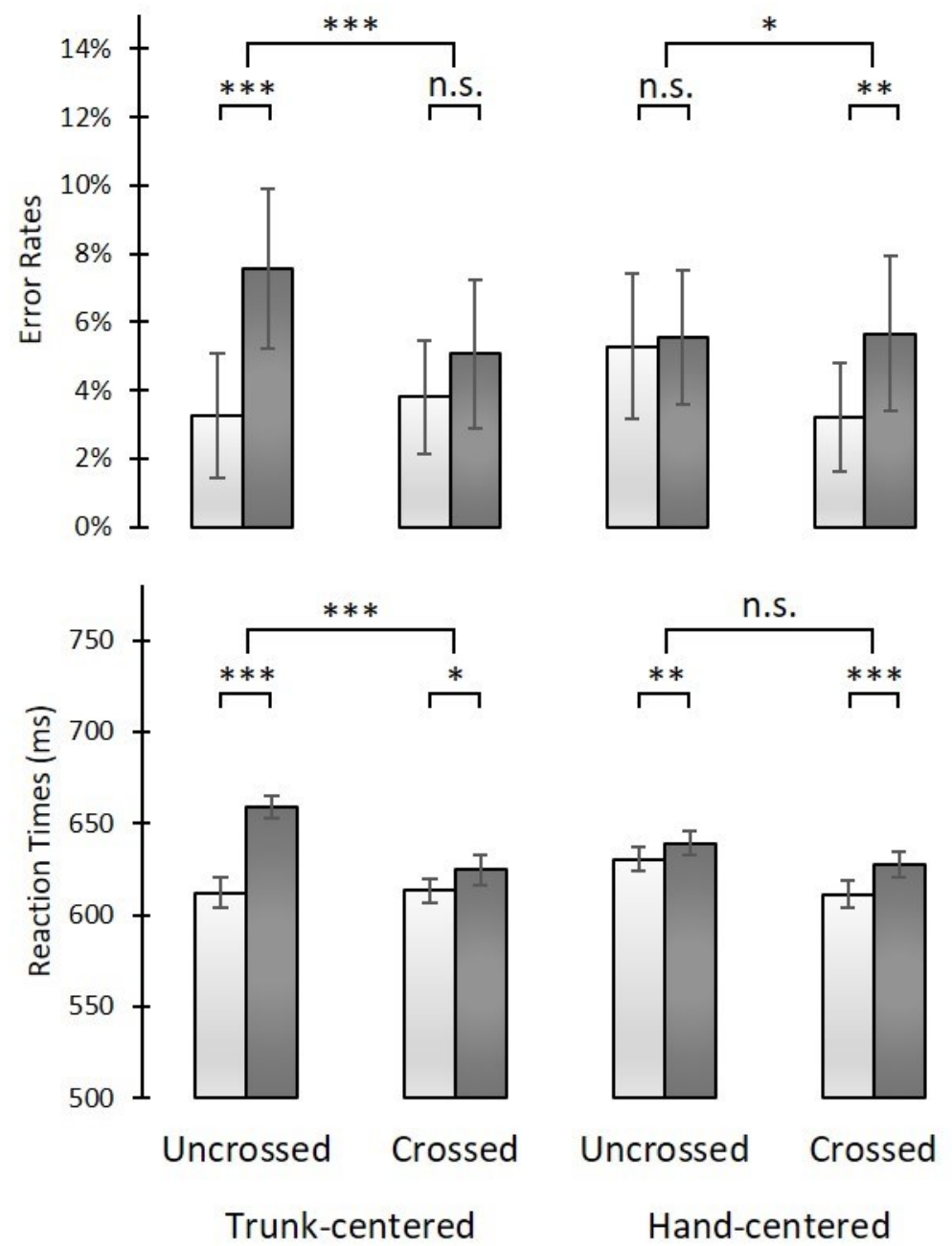

Trunk-centered Hand-centered 
Figure 1. Experiment 1. In this tactile Simon task, the stimulus was randomly presented to one of four possible locations, two on each hand (to the pinkie and thumb of the left and right hand), as indicated by the black arrows in the left panel. In different blocks of trials the hands were uncrossed or crossed. The right panel shows error rates (line graphs) and response times (bar graphs) separately for the trunkcentered Simon effect, hand-centered Simon effect, and the different hand postures (uncrossed vs. crossed hands). Error bars indicate $95 \%$ within-subjects confidence intervals. Number of asterisks denotes P-valuesp-values $\left(* \mathrm{P}<0.05,{ }^{* *} \mathrm{P}<0.01,{ }^{* * *} \mathrm{P}<0.001\right)$ obtained in the ANOVAs.

\subsection{Discussion}

The results from Experiment 1 demonstrated that the tactile Simon effect can occur in multiple frames of reference - both in a "somatotopic" frame of reference (as observed in Medina et al., 2014) and a "hand-centered" frame of reference, in which stimulus codes can also be generated based on whether the stimulus is on the left- or right-side of the hand. In this paradigm, we found both a significant somatotopic Simon effect and a significant "hand-centered" effect. But surprisingly, we found a significant decrease in the somatotopic Simon effect with the arms crossed versus uncrossed. This strongly contrasts previous findings in which the somatotopic Simon effect is observed in either body posture. This finding will be discussed further in the general discussion.

\section{Experiment 2: Is the somatotopic Simon effect obligatory?}

In Experiment 1, we found both a somatotopic and a hand-centered Simon effect, providing evidence that tactile stimulus codes could be generated based on multiple frames of reference. Tactile stimuli presented to each hand are represented in the contralateral somatosensory cortex. Primary somatosensory representations code stimulus location in a somatotopic frame of reference, and do this regardless of task - i.e. stimulation of the left index finger will always result in activation in right somatosensory cortex. If stimulus codes are generated at this level, then one possibility is that there will always be a somatotopic Simon effect, regardless of the task. However, a second possibility is that somatotopic stimulus codes are not always generated, leading to conditions in which a somatotopic Simon effect would not be observed.

In Experiment 2, participants performed two tactile Simon tasks. One was similar to the one used in Experiment 1 (with uncrossed hands) in which the tactile stimulus was randomly presented to one of four possible locations, two on each hand (the two hands task, see Figure 2). In the other tactile Simon 
task the stimulus was presented to one of two possible locations on the same hand (pinky or thumb) during any given block (the one hand task), with the left and right hands tested in different blocks of trials. In this version of the task, there would be no need to code the stimulated hand during a block, as all stimuli are presented to one hand only. Given that no stimuli are being presented to the other hand, coding in a somatotopic frame of reference may not be relevant. If so, we would expect no somatotopic Simon effect, but would predict a hand-centered Simon effect. However, the alternative is an "obligatory" somatotopic Simon effect in which somatotopic stimulus codes are generated regardless of whether one or two hands are tested. In this case, one would predict a significant somatotopic Simon effect.

\subsection{Methods}

\subsubsection{Participants}

Existing literature suggests that the tactile Simon effect is characterised by a large to very large effect size (e.g. dz $=1.42$ in Ruzzoli \& Soto-Faraco, 2017; Medina et al., 2014). Furthermore, studies investigating the Simon effect relative to the hemifield and relative position of visual stimuli have suggested that both these effects are of similar size (e.g. Lambert, Tavernier \& D'Ydewalle, 1992). Finally, both visual and tactile Simon effects have comparable effect sizes (e.g. Ruzzoli \& Soto-Faraco, 2017). Based on these considerations we tested a sample of 24 which is sufficient to detect an effect size of $(\mathrm{dz}=.6)$ with $80 \%$ power, and an alpha of .05 (two-tailed), as calculated with $\mathrm{G} *$ Power 3.1.9.2. for ttests (Means: difference between two dependent means, matched pairs).

Twenty-five participants took part in the study. One was excluded because they were unable to complete the task. Therefore, a total of 24 participants (three left-handed, 19 females) remained in the sample (mean age 20.4 years, age range 18-33). The study was approved by the Psychology research Ethics Committee of the University of Edinburgh.

\subsubsection{Apparatus, stimuli and procedure}

Participants were seated with both hands resting in their typical side of space, palms-down (with fingers marginally separated) on separate pieces of flat foam positioned on a table. A white pin fixed to the foam support indicated the expected position of participants' index fingers so that the distance between the left and right index fingers was $50 \mathrm{~cm}$ ( $25 \mathrm{~cm}$ to the table and body midline). A sheet of black cardboard obscured the participants' hands from view and a white pin located in the middle of this panel (38 $\mathrm{cm}$ in front of them on the midline) served as a fixation point (see Figure 1). 
To deliver the tactile stimulation miniature solenoid tappers (MST5S M \& E Solve, Rochester) were attached to the lateral surfaces of the distal segments of the thumb and little finger on both hands with Velcro straps. These solenoids propelled a blunt conical rod onto the skin when a current was passed through them. They were controlled by a Miniature Solenoid Tapper Controller (Nk3 (MSTC3-4), M \& E Solve, Rochester) connected to a Desktop Dell OptiPlex 745 computer (Dell Inc., Round Rock, TX) running an E-Prime 2.0 (Psychology Software Tools Inc., Sharpsburg, PA) script. To mask any potential noise by the solenoids, participants listened to white noise through headphones (Sony MDR-V150 Dynamic Stereo Headphones Sony Corporation, Tokyo). Two foot pedals were used to record responses via a PST Serial Response Box Model 200a (Psychology Software Tools Inc., Sharpsburg, PA). Participants placed their feet (without shoes) on the left and right pedal respectively (17 cm distant, 8.5 $\mathrm{cm}$ from body midline) and depressed either one of them following task instructions.

Tactile stimuli consisted of high $(25 \mathrm{~Hz})$ or low $(12.5 \mathrm{~Hz})$ frequency vibrations of $240 \mathrm{~ms}$ duration. For both high and low frequency vibrations the solenoids made contact with the skin during $5 \mathrm{~ms}$ pulses. Successive pulses were interleaved by offset intervals during which no contact was made between the solenoid and the skin. These inter-pulse intervals were $35 \mathrm{~ms}$ long for the high frequency stimuli (on-off cycle repeated 6 times) and 75ms long for the low frequency ones (on-off cycle repeated 3 times).

Each trial began with the presentation of a tactile stimulus. Following stimulus offset an interval of $1560 \mathrm{~ms}$ was used to record responses and the trial ended $1900 \mathrm{~ms}$ after stimulus onset.

Each participant completed eight blocks of 80 trials each (640 trials in total). Each block consisted of 10 repetitions of the eight possible combinations of stimuli (i.e. left or right hand, left or right finger within each hand and high or low frequency stimulus). On four consecutive blocks, both hands were placed on the table and the tactile stimulus could be presented to one of the four possible stimulus location (thumb or little finger of the left and right hand: Two hands task). In the remaining 4 blocks, one hand was placed on the table (while the other was resting on the ipsilateral leg under the table) and stimuli were presented to one of the two possible stimulus locations on that hand (thumb or little finger of the left or right hand: one hand task). The task relevant hand was changed after two consecutive blocks of 80 trials. Task order (one hand task followed by two hands task or vice-versa) was counterbalanced across participants, as was the order of the task relevant hand in the one hand task.

A 2 (task) x 2 (trunk-centered congruency) x 2 (hand-centered congruency) factorial design was used to investigate whether the location of tactile stimuli was automatically coded with respect to both 
the stimulated hand and to the stimulated finger within that hand. The variable trunk-centered congruency coded the spatial relationship (congruent vs. incongruent) between the stimulated hand (left or right hand, regardless of which finger received the stimulation) and the responding foot (left or right foot). The variable hand-centered congruency coded the spatial relationship (congruent vs. incongruent) between the stimulated finger (left or right finger, regardless of which hand received the stimulation) and the responding foot (left or right foot). Finally, the variable task (one vs. two hands task) indicated the number of hands that were task-relevant in a given block of trials.

Participants were instructed to respond as quickly and accurately as possible to the frequency of the tactile stimulus regardless of its location, by pressing the left or right foot pedals according to stimulus-response instructions. The frequency-to-foot-pedal mapping was counterbalanced across participants. Half of the participants responded with the left foot pedal for a high frequency stimulus and the right pedal for a low frequency stimulus. The opposite stimulus-response mapping was assigned to the other half of participants.

Each participant was instructed as to their mapping and received pre-experiment training to ensure that they could differentiate between high- and low-frequency stimuli. First, they were given examples of high- and low-frequency tactile stimuli, set at the same intensity and durations as those presented in the experiment. When participants struggled to differentiate the two frequencies, the experimenter verbally labelled them until they were able to discriminate the stimuli accurately. Participants completed a 40 trial training session. If the overall accuracy was lower than $70 \%$ the training was repeated. The mapping and hand position adopted for the training period were the same as in the first block of experimental trials.

\subsection{Results}

We excluded $1.82 \%$ of trials that were outliers (RT values $>3 S D$ s above or below the participant's mean RT for the block), anticipatory responses before the stimulus was presented, or other input errors.

\subsubsection{Reaction times}

Reaction times on correct trials were log-transformed and submitted to a 2 (task: one hand vs. two hands) x 2 (trunk-centered congruency) x 2 (hand-centered congruency) repeated measures ANOVA. A 19 ms trunk-centered congruency effect was observed, $\mathrm{F}(1,23)=46.3, p<.001, \eta_{p}^{2}=0.67$, with congruent responses $(679 \mathrm{~ms})$ faster than incongruent ones $(698 \mathrm{~ms})$. In addition, a $15 \mathrm{~ms}$ hand-centered congruency effect was also observed, $\mathrm{F}(1,23)=8.01, p=.009, \eta_{p}^{2}=0.26$ with congruent responses 
faster than incongruent ones (681 ms vs. $696 \mathrm{~ms}$, respectively). Last, there was a significant interaction between trunk-centered congruency and task, $\mathrm{F}(1,23)=9.25, p=.006, \eta_{p}^{2}=0.29$. Follow-up analyses conducted separately for each task revealed the presence of a $31 \mathrm{~ms}$ significant trunk-centered congruency effect in the two hands task, $\mathrm{F}(1,23)=46.53, p<.001, \eta_{p}^{2}=0.67(679 \mathrm{~ms}$ and $711 \mathrm{~ms}$ for congruent and incongruent trials respectively). In contrast, there was no significant trunk-centered congruency effect in the one hand task, $\mathrm{F}(1,23)=2.58, p=.121$ ( $7 \mathrm{~ms}$ trunk-centered congruency effect, $679 \mathrm{~ms}$ and $686 \mathrm{~ms}$ for congruent and incongruent, respectively).

Reaction time data (log-transformed) were also analysed in a linear mixed model using the lmertest package in R 3.5.1. with task, trunk-centered somatotopic congruency, and hand-centered external congruency as fixed effects. The following random slope intercepts were also included: (1+task|subject), (1+trunk- centered somatotopic congruency|subject), (1+external hand-centered congruency|subject). We found a significant main effect of hand-centered external congruency $(b=.02, t=2.9, p=.008)$ and of trunk-centered somatotopic congruency $(b=.03, t=7.07, \mathrm{p}<.001)$. In addition, we observed a significant interaction between task and trunk-centered somatotopic congruency $(b=.004, t=5.1, p<$ .001). A significant trunk-centered Simon effect was present in the two hands task $(b=.05, t=6.48, p<$ $.001)$, but not in the one hand task $(\mathrm{t}=1.99, \mathrm{p}=.059)$. The interaction between task and hand-centered congruency $(b=-.02, t=-2.46, p=.014)$ revealed that the hand-centered Simon effect was present in the one hand task $(b=0.03, t=3.79, p<.001)$ but not in the two hands task $(t=1.4, p=0.18)$. No other comparisons were significant.

\subsubsection{Accuracy}

Errors were analysed with a generalised linear mixed-effects model (GLMM), with the same fixed effects and random effects structure as in the reaction time linear mixed model in 3.2.1.. Participants made less errors on trunk-centered congruent than incongruent trials $(8.5 \%$ and $12.1 \%$, respectively; $b=$ $0.44, \mathrm{z}=4.16, p<.001)$. Moreover, participants responded more accurately on hand-centered congruent than incongruent trials $(9.3 \%$ and $11.2 \%$, respectively; $\mathrm{b}=0.18, \mathrm{z}=2.31, p=.021)$. A significant interaction between hand-centered and trunk-centered congruency $(\mathrm{b}=0.25, \mathrm{z}=2.27, p=.023)$ revealed that the trunk-centered Simon effect was stronger on incongruent hand-centered trials (error rate difference: $5.1 \%$ ) than it was on congruent ones (error rate difference: $2.2 \%$ ). Results also revealed an interaction between hand-centered congruency and task $(b=-0.27, z=-2.48, p=.013)$, such that the 
hand-centered Simon effect was stronger in the one hand task (2.7\%) than in the two hands task (1.1\%).. The interaction between trunk-centered congruency and task was also significant $(\mathrm{b}=0.25, \mathrm{z}=2.20, p=$ .028 ), with a stronger trunk-centered Simon effect in the two hands task (4.8\%) than in the one hand task $(2.5 \%)$. Finally, a three-way interaction between trunk-centred congruency, hand-centred congruency, and task was observed $(\mathrm{b}=0.46, \mathrm{z}=2.07, p=.038)$. This reflected the presence of a significant interaction between hand-centered congruency and trunk-centered congruency in the two-hands task $(b=0.49, z=$ $3.22, p=.001)$ but not in the one-hand task $(\mathrm{b}=0.03, \mathrm{z}=0.2, p=.837)$.

Error rates were also submitted to a 2 (task) x 2 (trunk-centered congruency) $\times 2$ (hand-centered congruency) repeated measures ANOVA. A main effect of trunk-centered congruency was observed, $\mathrm{F}(1,23)=15.11, p<.001, \eta_{\mathrm{p}}^{2}=0.4$, indicating that trunk-centered congruent responses were more accurate than incongruent ones (error rates: $8.5 \%$ and $12.1 \%$, respectively). A hand-centered congruency main effect was also observed, $\mathrm{F}(1,23)=7.56, p=.011, \eta_{\mathrm{p}}^{2}=0.25$, indicating that hand-centered congruent responses were more accurate than incongruent ones (9.3\% and $11.2 \%$, respectively). Last, a significant interaction between trunk-centered congruency and hand-centered congruency emerged to be significant, $\mathrm{F}(1,23)=6.09, p=.021, \eta_{\mathrm{p}}^{2}=0.21$, reflecting a smaller trunk-centered Simon effect on congruent hand-centered trials than on incongruent ones (2.2\% and 5.1\%, respectively). 


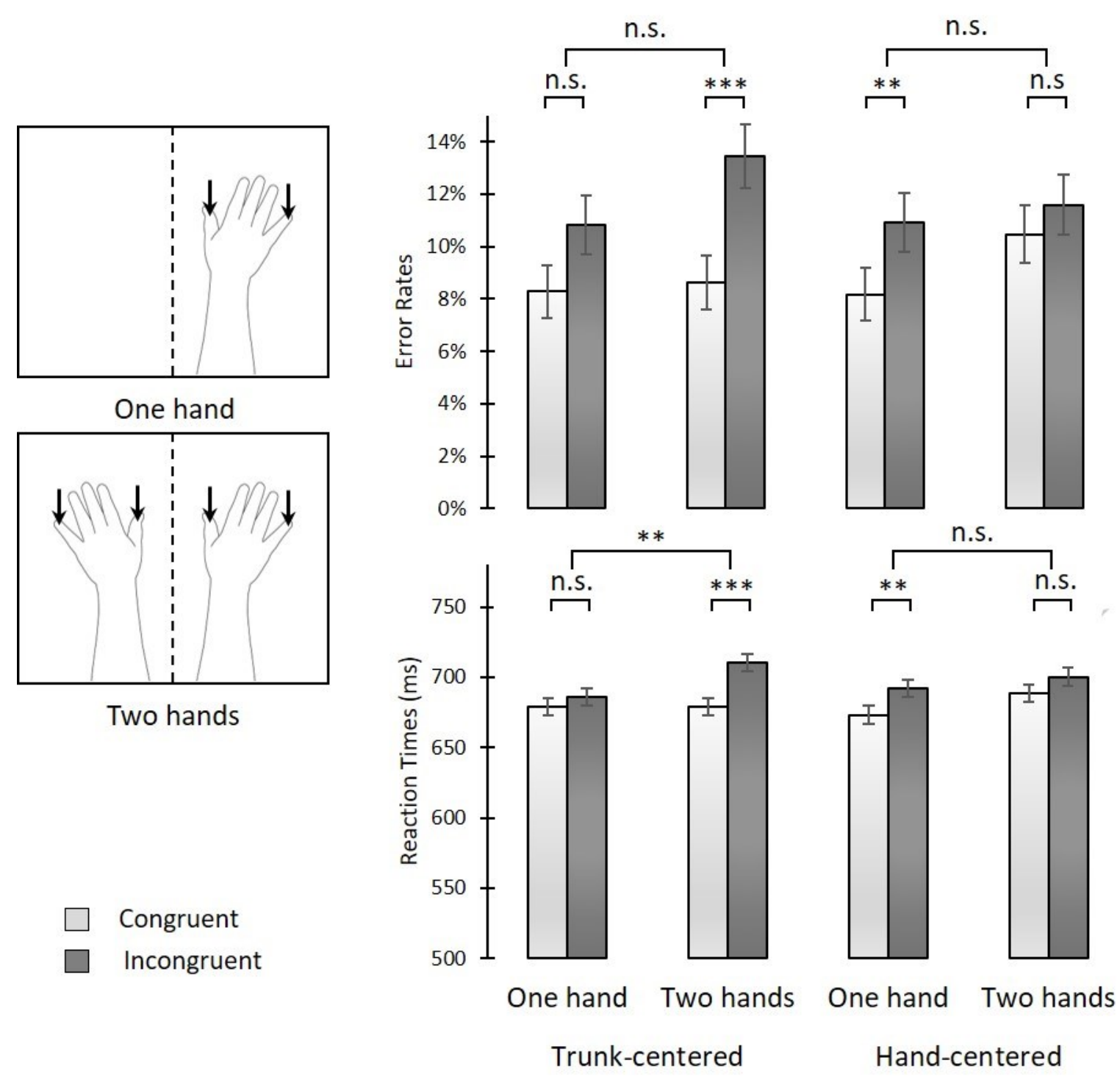

Figure 2. Experiment 2. Participants completed two different tactile Simon tasks in different blocks of trials. In the Two hands task, the tactile stimulus was randomly presented to one of four possible locations, two on each hand, as indicated by the black arrows in the left panel. In the One hand task, the stimulus was randomly presented to one of two possible locations on the same hand, and the different hands were tested on different blocks of trials. The right panel shows error rates (line graphs) and response times (bar graphs) separately for the trunk-centered Simon effect and for the hand-centered Simon effect. Error bars indicate $95 \%$ within-subjects confidence intervals. Number of asterisks denotes P-valuesp-values $(* \mathrm{P}<0.05, * * \mathrm{P}<0.01, * * * \mathrm{P}<0.001)$ obtained in the ANOVAs. 


\subsection{Discussion}

The results of Experiment 2 provide additional evidence that the trunk-centered Simon effect is modulated by specific task requirements. Specifically, when only one hand is used the trunk-centered spatial code is not generated. This suggests that the coding of the tactile stimuli based on the somatotopic trunk-centered reference frame is not automatic.

\section{Experiment 3: How are left and right assigned in the hand-centered tactile Simon effect?}

Experiments 1 and 2 demonstrated the existence of hand-centered congruency effects when stimuli were presented to the same hand. The aim of the following studies is to assess the reference frame upon which this spatial code is based. Experiment 3 aimed to establish whether the within hands automatic spatial code is modulated by hand posture. The hands were tested individually (one hand task, two possible stimulus locations thumb and little finger), and hand posture (palm up or down / supinated or pronated) was manipulated. The aim of the hand posture manipulation (palm up and palm down) was to examine left-right assignment for stimulus codes generated in the hand-centered Simon effect. One possibility is that left and right are inherited from the participant's trunk. In this coordinate system, the pinky of the left hand would be coded as "left" with the palm down, whereas it would be coded as "right" with the palm up. A second possibility is that left and right are assigned based on a somatotopic representation, where left-right assignment is fixed regardless of hand/body orientation. For example, in a somatotopic representation, the pinky side of the left hand could always be encoded as the left side of the hand, with the thumb side represented as the right side of the hand. If this is the case, then stimuli presented to the left pinky finger will always generate a "left" stimulus code, regardless of hand orientation. For the hand-centered Simon effect, we predict that left and right will be encoded based on an external coordinate system. In Experiment $3 \mathrm{a}$ the task-relevant hand was aligned with the trunk/body midline, while in Experiment $3 \mathrm{~b}$ it was placed on the side $(25 \mathrm{~cm}$ to the left or right of the body midline; right hand on the right side and left hand on the left side on different blocks of trials).

\subsection{Experiment 3a}

\subsubsection{Methods}

All research for Experiment 3 was conducted at the University of Delaware with approval from the local Institutional Review Board. Furthermore, all research protocols were preregistered on Open Science Framework at https://osf.io/dw52r/. 


\subsubsection{Participants}

As stated in Experiment 1, our recommended sample size was 32 subjects. However, for this experiment, we also wanted to ensure that our sample size was sufficient to find that there was no somatotopic Simon effect with only one hand. To obtain $80 \%$ power for an equivalence test with an equivalence bound of .7 and an $\alpha$ of .05, the recommended sample size is 36 (see Lakens, 2017).

Forty-one participants were tested. As noted in our preregistration plan, we only included the first 36 participants that met inclusion criteria for our analysis (mean age: 18.6, range: 18-22, 30 females, four left-handed). No participants were excluded due to experimental issues. All participants were undergraduates at the University of Delaware, who were reimbursed via participation credits for an Introduction to Psychology course.

\subsubsection{Apparatus, Stimuli and Procedure}

The apparatus, stimuli and procedure, including the pre-screening procedure, were all the same as in Experiment 1, except for the following differences. For each block, the participant was told to place either their left or right hand on the table, with their middle finger aligned with the midline of their trunk and the monitor. The hand that was not on the table was positioned on their lap. Stimuli were only presented to the hand that was on the table.

Each participant was presented with four blocks, in which hand posture (palm up, palm down) and hand (left or right) was modified, with one block per combination. Blocks were presented in a randomized order. Each block consisted of 16 practice trials followed by 96 experimental trials, counter-balanced for stimulus location and intensity. Stimulus-response mapping (A: left foot pedal, high-intensity tactile stimulus; right foot pedal, low-intensity tactile stimulus; B: left foot pedal, low-intensity tactile stimulus; right foot pedal, high-intensity tactile stimulus) was balanced across subjects (i.e. half had mapping A, half had mapping B).

We excluded $1.14 \%$ of trials that were outliers (RT values $>3$ SDs above or below the participant's mean RT for the block), anticipatory responses before the stimulus was presented, failures to respond, or other input errors. All participants passed the pre-screener.

\subsubsection{Results}

\subsubsection{Reaction times}

Reaction times were log-transformed and analyzed in a 2 (Hand Posture) x 2 (Trunk- Centered Somatotopic Congruency) x 2 (Hand-Centered External Congruency) analysis of variance (ANOVA). 
First, there was a main effect of trunk-centered somatotopic congruency, as participants were $6 \mathrm{~ms}$ faster for trunk-centered somatotopically congruent trials $(622 \mathrm{~ms})$ than for trunk-centered somatotopically incongruent trials $(628 \mathrm{~ms}), F(1,35)=6.315, p=.017, \eta_{p}^{2}=0.15$. We also observed a significant external hand-centered congruency effect, as participants were $39 \mathrm{~ms}$ faster for externally congruent trials (606 $\mathrm{ms})$ compared to externally incongruent trials (645 ms), $F(1,35)=164.45, p<.001, \eta_{p}^{2}=0.83$. Note that if there was a significant hand-centered effect in which left and right were encoded somatotopically, then we would observe a hand posture by hand-centered external congruency interaction. However, this was not the case, $F(1,35)=2.32, p=.137, \eta_{p}^{2}=.062$. Finally, there was a significantly three-way interaction between hand posture, trunk-centered somatotopic congruency, and hand-centered external congruency, $F(1,35)=5.19, p=.029, \eta_{p}^{2}=0.13$. With palms up, there was a stronger hand-centered Simon effect on trunk-centered congruent $(41 \mathrm{~ms})$ versus incongruent $(27 \mathrm{~ms})$ trials. With the palms down, the opposite pattern emerged, with a larger hand-centered Simon effect for trunk-centered incongruent $(50 \mathrm{~ms})$ versus congruent (41 ms) trials.

Based on our preregistration document, we also analysed reaction time data (log-transformed) in a linear mixed model using the lmertest package in $\mathrm{R}$ 3.5.1. with hand position, trunk-centered somatotopic congruency, and hand-centered external congruency as fixed effects. The following random slope intercepts were also included: (1+hand posture|subject), (1+trunk- centered somatotopic congruency|subject), (1+external hand-centered congruency|subject). As in the ANOVA, we found a significant main effect of hand-centered external congruence $(b=-.062, t=-12.9, p<.001)$, trunkcentered somatotopic congruency $(b=-.009, t=-2.42, p=.020$ ), and a three-way hand position by trunkcentered somatotopic congruency by external congruency interaction $(b=-.034, t=-2.22, p=.032)$. No other comparisons were significant, including the hand posture by hand-centered external congruency interaction $(\mathrm{b}=.013, \mathrm{t}=1.48, \mathrm{p}=.148)$.

\subsubsection{Accuracy}

Errors were analyzed in a generalized linear mixed model (GLMM), with the same fixed and random effects structure as in the reaction time linear mixed model in 4.1.2.1. First, there was a main effect of external hand-centered congruence $(b=.875, \mathrm{z}=5.139, \mathrm{p}<.001)$, as participants made

significantly more errors on external hand-centered incongruent (5.6\%) versus external hand-centered congruent trials $(2.7 \%)$. There was also a trunk-centered somatotopic congruency by external hand- 
centered congruency $(\mathrm{b}=.647, \mathrm{z}=2.243, \mathrm{p}=.025)$, with the trunk-centered somatotopic Simon effect being greater on externally congruent trials $(1.2 \%)$ than in externally incongruent trials $(.3 \%)$. We did not find a significant hand posture by hand-centered external congruency interaction $(b=.068, z=.21$, $\mathrm{p}=.837)$.

Accuracy was also examined with a 2 (Hand Posture) x 2 (Trunk-Centered Somatotopic Congruency) x 2 (Hand-Centered Congruency) analysis of variance (ANOVA). While there was a significant main effect of external hand-centered congruency, $F(1,35)=20.73, p<.001, \eta_{p}^{2}=0.37$, the trunk-centered somatotopic congruency by external hand-centered congruency interaction was not significant, $F(1,35)=3.34, p=.076, \eta_{p}^{2}=.087$.

\subsection{Experiment 3b}

\subsubsection{Methods}

\subsubsection{Participants}

The hand-centered Simon effect observed in the one hand task of Experiment 2 was characterised by an effect size of $d z=.66$. The total recommended sample to detect a similar effect with $80 \%$ power, and an alpha of .05 (two-tailed) was 21 using G*Power 3.1.9.2. for t-tests (Means: difference between two dependent means, matched pairs).

Twenty-one new participants at the University of Edinburgh were tested (one left-handed, 10 females, mean age 20.7 years, age range 18-24). The study was approved by the Psychology Research Ethics Committee of the University of Edinburgh.

\subsubsection{Apparatus, Stimuli and Procedure}

The apparatus, stimuli and procedure were identical to those used in Experiment 2 for the one hand task with the following exceptions. In the present experiment, hand posture (palm up vs palm down) was manipulated in different blocks of trials. As in Experiment 2, one hand task, tactile stimuli were randomly presented to the thumb or little finger of one hand. Left and right hands were tested in different blocks of trials. The stimulated hand was always positioned laterally $25 \mathrm{~cm}$ from the midline within its natural hemi-space (left hand in the left hemi-space and right hand in the right one).

Each participant completed four blocks of 80 trials for the palm down as well as for the palm up hand posture. The stimulated hand was changed every two blocks, so that each successive set of two blocks used the same hand in the same hand posture. The order of these conditions was counterbalanced 
across participants, so that half of them started with the left and half with the right hand, and half with the palm up or palm down posture.

\subsubsection{Results}

We excluded $1.74 \%$ of trials that were outliers (RT values $>3$ SDs above or below the participant's mean RT for the block), anticipatory responses before the stimulus was presented, or response omissions.

\subsubsection{Reaction times}

Reaction times were log-transformed and submitted to a 2 (hand posture) x 2 (trunk-centered somatotopic congruency) x 2 (hand-centered external congruency) repeated measures ANOVA. The

presence of a main effect of hand-centered congruency, $\mathrm{F}(1,20)=91.25, p<.001, \eta_{p}^{2}=0.82$, revealed a $31 \mathrm{~ms}$ external hand-centered Simon effect with faster responses on congruent than incongruent trials (534 ms vs. $565 \mathrm{~ms}$, respectively). The external hand-centred congruency effect was not modulated by hand posture, $\mathrm{F}(1,20)=1.4, p=.250$, as confirmed by the fact that reliable hand-centered external Simon effects were observed when the hands were palm down $(37 \mathrm{~ms}), \mathrm{F}(1,20)=52.1, p<.001, \eta_{p}^{2}=0.72$, as well as palm up $(24 \mathrm{~ms}), \mathrm{F}(1,20)=31.1, p<.001, \eta_{p}^{2}=0.61$. This suggests that the representation of tactile stimuli was based on an external hand-centered reference frame. There was no significant main effect of trunk-centred congruency, $(\mathrm{F}(1,20)=1.4, p=.250$, ) and no trunk-centred congruency by hand posture interaction, $\mathrm{F}(1,20)=0.42, \mathrm{p}=.052$. In addition, results revealed the presence of a significant three-way interaction, $\mathrm{F}(1,20)=20.99, p<.001, \eta_{p}^{2}=0.51$. Follow-up analyses conducted separately for each hand posture revealed the presence of significant interactions between hand-centred and trunkcentred congruency in both the palm down, $\mathrm{F}(1,20)=8.2, p=.001, \eta_{p}^{2}=0.29$, and palm up conditions, $\mathrm{F}(1,20)=12.76, p<.001, \eta_{p}^{2}=0.39$. With the palm down the hand-centered Simon effect was smaller for trunk-centered congruent $(30 \mathrm{~ms} ; 529 \mathrm{~ms}$ and $559 \mathrm{~ms}$ for hand-centered congruent and incongruent, respectively) than for trunk-centered incongruent trials (44 ms; $523 \mathrm{~ms}$ and $567 \mathrm{~ms}$ for hand-centered congruent and incongruent respectively). In contrast, with the palm up the hand-centered Simon effect was larger for trunk-centered congruent trials (33 ms; $536 \mathrm{~ms}$ and $569 \mathrm{~ms}$ for hand centered congruent and incongruent, respectively) than for trunk-centered incongruent ones (14 ms; $550 \mathrm{~ms}$ and $564 \mathrm{~ms}$ for hand centered congruent and incongruent, respectively).

Reaction time data (log-transformed) were also analysed in a linear mixed model (GLMM) using the lmertest package in R 3.5.1. with hand posture, trunk-centered somatotopic congruency, and handcentered external congruency as fixed effects. The following random slope intercepts were also included: 
(1+hand posture|subject), (1+trunk- centered somatotopic congruency|subject), (1+external handcentered congruency|subject). We found a significant main effect of hand-centered external congruence $(b=0.06, t=9.85, p<.001)$. A significant interaction between hand posture and hand-centered external congruency $(b=-.02, t=-3.43, p<.001)$, revealed that the hand-centered external Simon effects observed with palm down $(\mathrm{b}=.07, \mathrm{t}=7.41, \mathrm{p}<.001)$ was stronger than that observed with palm up $(\mathrm{b}=.04, \mathrm{t}=$ $6.14, \mathrm{p}<.001)$ hand postures. In addition, results revealed a significant three-way interaction $(\mathrm{b}=-.06, \mathrm{t}$ $=-4.55, \mathrm{p}<.001)$. Separate analyses carried out for each hand posture revealed significant interactions between trunk-centered and hand-centered congruency for both the palm down $(b=.03, t=2.87, p<$ $.004)$ and the palm up postures $(b=-.03, t=-3.57, \mathrm{p}<.001)$.

\subsubsection{Accuracy}

Error rates were analysed in a generalised linear mixed-effects model (GLMM), with the same fixed and random effects structure as in the reaction time linear mixed model in 4.2.2.1. Fewer errors were observed on hand-centered congruent than incongruent trials $(3.8 \%$ and $6.6 \%$, respectively, $\mathrm{b}=0.69, \mathrm{z}$ $=4.21, p<.001)$. The trunk-centered congruency by hand posture interaction $(\mathrm{b}=0.38, \mathrm{z}=2.31, p=$ .021) revealed that the trunk-centered Simon effect was greater in the palm down versus palm up posture ( $1.2 \%$ and $0 \%$, respectively), although neither of these congruency effects were statistically significant (palm down: $\mathrm{z}=-0.93 p=.350$; palm up: $\mathrm{z}=1.79 p=.075$ ). Last, results revealed a significant threeway interaction between hand posture, trunk-centred congruency and hand-centred congruency $(b=1.13$, $\mathrm{z}=3.45, p<.001)$. Significant interactions between trunk-centered and hand-centered congruency were observed in the palm down $(\mathrm{b}=0.62, \mathrm{z}=0.23, p=.007)$ and palm up posture $(\mathrm{b}=-0.54, \mathrm{z}=-2.25, p=$ .025). With palm down the hand-centered congruency effect was larger on trunk-centered incongruent trials $(4.3 \%$ hand-centered SE) than on trunk-centered congruent trials $(1.5 \%)$. In contrast, in the palm up posture, the hand-centered congruency effect was larger on trunk-centered congruent trials $(3.6 \%$ hand-centered SE; ) than on trunk-centered incongruent ones ( $2 \%$ hand-centered SE).

Error rates were also submitted to a 2 (hand posture) x 2 (trunk-centered congruency) x 2 (handcentered congruency) repeated measures ANOVA. There were fewer errors on hand-centered congruent than incongruent trials $(3.8 \%$ and $6.6 \%$, respectively; main effect of hand-centered congruency, $\mathrm{F}(1,20)$ $=13.49, p=.002, \eta_{\mathrm{p}}^{2}=0.4$. Furthermore, the three-way interaction emerged to be significant, $\mathrm{F}(1,20)=$ $4.53, p=.046, \eta_{\mathrm{p}}^{2}=0.18$. Follow-up analyses were conducted separately for each hand posture. In the 
palm down posture, a significant interaction between hand-centered and trunk-centered congruency, $\mathrm{F}(1$, 20) $=5.87, p=.025, \eta_{p}^{2}=0.3$, revealed that the hand-centered Simon effect was present on trunk-centered incongruent trials $(4.3 \% ; \mathrm{t}(33.42)=4, p=.002)$ but not on trunk-centered congruent ones $(1.5 \% ; \mathrm{t}(33.42)$ $=-1.45, p=.476)$. With the palm up, there was no interaction between hand-centered congruency and trunk-centered congruency $(\mathrm{F}(1,20)=1.24, p=.278)$.

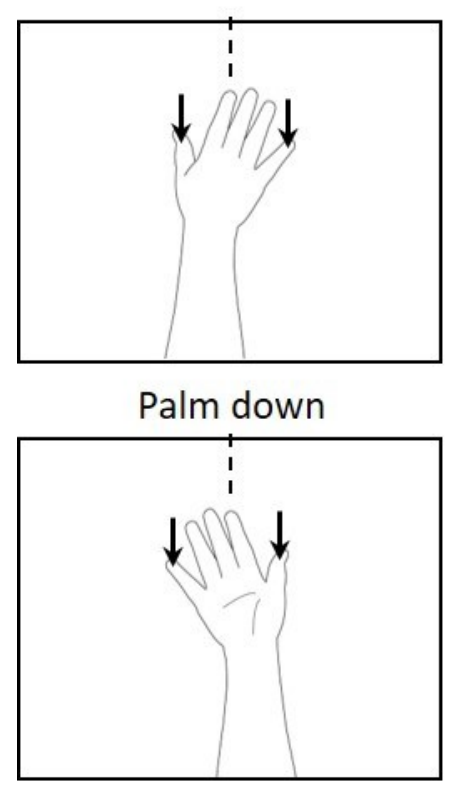

Palm up
Congruent Incongruent
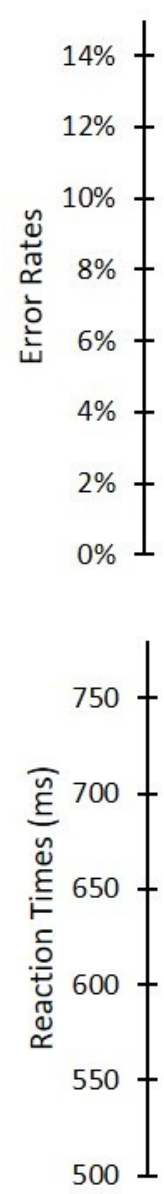

Patr

Palms down Palms up

Hand-centered $3 \mathrm{~A}$
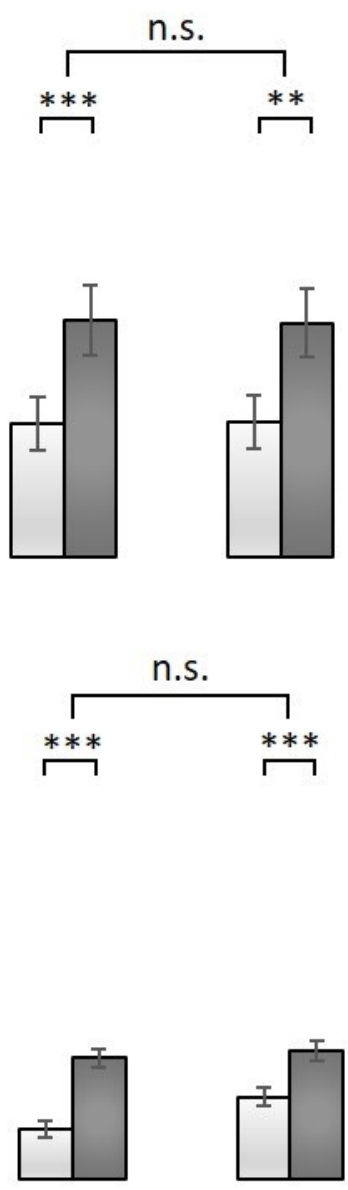

Palms down Palms up

Hand-centered 3B

Figure 3. Experiment 3. In this one hand tactile Simon task, the stimulus was randomly presented to one of two locations on the same hand, as indicated by the black arrows in the left panels. In different blocks of trials the left or right hand was positioned with the palm up or with the palm down. In Experiment 3a, the hand was aligned with the body midline, while in Experiment $3 \mathrm{~b}$ the hand was positioned on its ipsilateral side. The figure on the right shows error rates (line graphs) and response times (bar graphs) 
for the hand-centered Simon effect separately for the different hand postures (palm up vs. palm down). Error bars indicate $95 \%$ within-subjects confidence intervals. Number of asterisks denotes P-valuespvalues $(* \mathrm{P}<0.05, * * \mathrm{P}<0.01, * * * \mathrm{P}<0.001)$ obtained in the ANOVAs.

\subsection{Discussion}

The results of Experiment $3 \mathrm{a}$ and $3 \mathrm{~b}$ demonstrate that the hand-centered congruency effect is not modulated by hand posture, palms up or palms down. This provides evidence that the location of the tactile stimuli within the hand were coded according to an external reference frame.

\section{Experiment 4: Does hand position influence the hand-centered Simon effect?}

When participants keep their gaze on a central fixation point and this fixation is aligned with the mid-sagittal body midline (as in all studies reported here), gaze-centred, head-centered and trunkcentered reference frames coincide. Under these experimental conditions, the body midline can be used as a reference point to encode the locations of tactile stimuli presented to the hands according to an external reference frame. These external spatial codes change as a function of the distance between the hand and the body midline.

Here, we investigate whether the distance of the hand from the body midline (i.e. its position in external space) modulates the hand-centred Simon effect. Experiments $3 \mathrm{a}$ and $3 \mathrm{~b}$ demonstrated that the center of the hand is the likely reference point used to encode left-right assignments when tactile stimuli are presented to the same hand. It is possible that the hand-centered spatial codes are computed independently from the position of the hand with respect to the body midline. If this is the case, similar hand-centered Simon effects should be observed regardless of the hand distance from the body midline. However, it is also possible that the hand-centered spatial codes take into account - at least in part - also the position of the hand with respect to the body midline. If this is the case the size of the hand-centered Simon effect should be maximal when the hand is aligned with this midline and decrease as a function of its distance from it (when the hand is located on its own hemifield).

In this study, we investigated whether the hand-centered Simon effect is modulated by the location of the hand relative to the body midline. Each hand was tested separately (one hand task, two possible stimulus locations) and in different blocks it was located either in a central position (aligned with trunk 
midline) or in a lateral positions (25 $\mathrm{cm}$ to the left or right of the trunk midline, right hand in the right side and left hand in the left side).

\subsection{Methods}

\subsubsection{Participants}

In Experiment $3 b$ the effect size of the hand-centered Simon effect was very large, dz $=.19$. Although only 5 participants are sufficient for an effect of this size (with $80 \%$ power, an alpha of .05 (two-tailed; G*Power 3.1.9.2. for t-tests: Means: difference between two dependent means, matched pairs), we tested a similar sample used in previous studies to facilitate direct comparisons across studies. Twenty-three new participants were tested (three left-handed, 10 females) remained in the sample (mean age 20.7 years, age range 18-24). The study was approved by the Psychology research Ethics Committee of the University of Edinburgh.

\subsubsection{Apparatus, Stimuli and Procedure}

The apparatus, stimuli and procedure were identical to those used in Experiment 2 (one hand task) with the exception that the position of the stimulated hand was manipulated in different blocks of trials (hand central vs. hand lateral). Tactile stimuli were presented to one hand at a time. The hand was either positioned centrally (aligned with the body midline) or laterally $(25 \mathrm{~cm}$ from body midline, the right hand in the right hemi-space and the left hand in the left hemi-space). Participants completed two blocks of 80 trials each for the following conditions (all with palms facing down): left hand central, left hand lateral, right hand central, right hand lateral. The stimulated hand (left vs right) changed every two blocks and hand location (central vs lateral) was changed every four blocks. Therefore, each successive set of two blocks used the same hand in the same hand position. The order of these testing conditions was counterbalanced across participants.

\subsection{Results}

We excluded $1.72 \%$ of trials that were outliers (RT values $>3$ SDs above or below the participant's mean RT for the block), anticipatory responses before the stimulus was presented, or response omissions.

\subsubsection{Reaction times}

Reaction times were log-transformed and submitted to a 2 (hand position) x 2 (trunk-centered congruency) x 2 (hand-centered congruency) repeated measures ANOVA. Only a hand-centered congruency main effect was observed, $\mathrm{F}(1,22)=26.8, p<.001, \eta_{p}^{2}=0.55$, indicating that hand-centered congruent responses were faster than incongruent ones $(590 \mathrm{~ms}$ and $615 \mathrm{~ms}$, respectively, $25 \mathrm{~ms}$ 
congruency effect). No hand-centered congruency by hand position interaction was present, $(\mathrm{F}(1,22)=$ $1.31, p=.265)$. Significant hand-centered congruency effects were observed in the central hand position $(27 \mathrm{~ms}, \mathrm{t}(33.81)=-5.47, p<.001)$ as well as in the lateral hand position $(22 \mathrm{~ms}, \mathrm{t}(74.61)=-4.13, p<$ $.001)$. No other main effects or interactions were present.

Reaction time data (log-transformed) was also analysed in a linear mixed model using the lmertest package in R 3.5.1. with hand position, trunk-centered somatotopic congruency, and hand-centered external congruency as fixed effects. The following random slope intercepts were also included: (1+hand posture|subject), (1+trunk- centered somatotopic congruency|subject), (1+external hand-centered congruency(subject). As in the ANOVA, we found a significant main effect of hand-centered external congruence $(\mathrm{b}=-.05, \mathrm{t}=-5.62, \mathrm{p}<.001)$. There was also a two-way trunk-centered somatotopic congruency by hand-centered external congruency interaction $(b=-.02, t=-2.7, p=.007)$. Separate analyses revealed the presence of a trunk-centered simon effect on hand-centered incongruent trials $(b=$ $-.02, \mathrm{t}=-3.12, \mathrm{p}=.005)$ but not on hand-centered congruent ones trials $(\mathrm{t}=.03, \mathrm{p}=.977)$.

\subsubsection{Accuracy}

Error rates were analysed in a generalised linear mixed-effects model (GLMM), with the same fixed and random effects structure as in the reaction time linear mixed model in 5.2.1. Participants made less errors on hand-centered congruent than incongruent trials $5.7 \%$ and $8.7 \%$, respectively; handcentered congruency main effect, $\mathrm{b}=0.42, \mathrm{z}=2.66, p=.008$ ). The interaction between hand-centered congruency and trunk-centered congruency $(b=0.41, \mathrm{z}=2.96, p<.001)$ revealed that a reliable handcentered Simon effect was present on trunk-centered incongruent trials $(b=0.66, z=3.09, p=.002)$, but not on the trunk-centered congruent ones $(z=1.05, p=.3$; error rate differences: $4.5 \%$ and $1.5 \%$ respectively). In addition, the three-way interaction between trunk-centred congruency, hand-centred congruency, and hand position emerged to be statistically significant $(\mathrm{b}=-0.56, \mathrm{z}=-2.07, p=.039)$. This reflected the presence of the hand-centered congruency $x$ trunk-centered congruency interaction in the central hand position $(b=0.7, \mathrm{z}=0.19, p<.001)$, but not in the lateral hand position $(\mathrm{z}=0.86, p=.392)$.

Error rates were also submitted to a 2 (hand position) x 2 (trunk-centered congruency) x 2 (handcentered congruency) repeated measures ANOVA. Only a hand-centered congruency main effect was 
observed, $\mathrm{F}(1,22)=4.77, p=.040, \eta_{\mathrm{p}}^{2}=0.18$, indicating that hand-centered congruent responses were more accurate than incongruent ones (5.7\% and $8.7 \%$, respectively). This hand-centered congruency main effect was not modulated by hand position, $\mathrm{F}(1,22)=0.40, \mathrm{p}=.530$. No other main effects or interactions were observed.
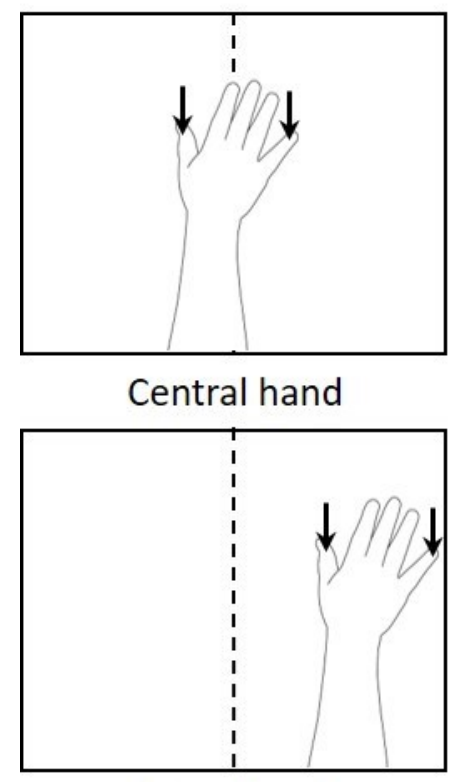

Lateral hand

Congruent

Incongruent
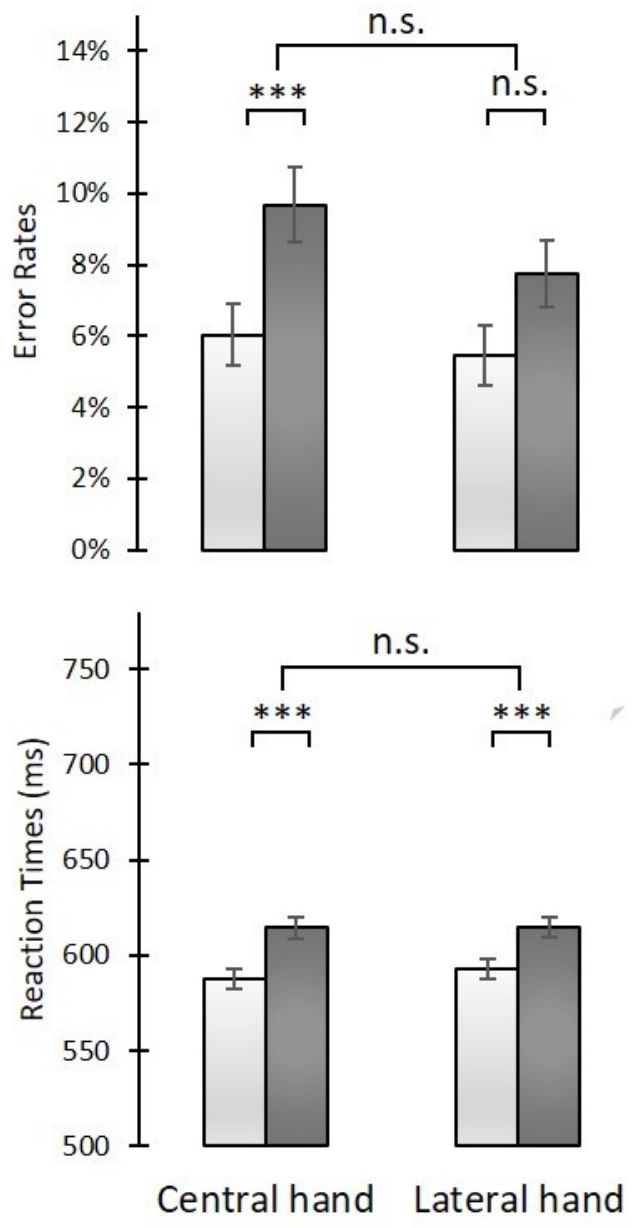

Hand-centered

Figure 4. Experiment 4. In this one hand tactile Simon task, the stimulus was randomly presented to one of two locations on the same hand, as indicated by the black arrows in the left panels. On different blocks of trials, the left or right hand was aligned with the body midline (central hand) or positioned to the side (lateral hand). The right figure shows error rates (line graphs) and response times (bar graphs) for the hand-centered Simon effect separately for the different hand postures (central vs lateral hand). Error bars 
indicate $95 \%$ within-subjects confidence intervals. Number of asterisks denotes P-valuesp-values $(* \mathrm{P}<0.05, * * \mathrm{P}<0.01, * * * \mathrm{P}<0.001)$ obtained in the ANOVAs.

\subsection{Discussion}

In line with previous studies, we observed a reliable hand-centred congruency effect in Experiment 4. However, it was not modulated by the position of the hand (central vs lateral), suggesting that hand position relative to the body midline does not impact the way in which tactile stimuli are spatially coded relative to the hand. While the location of the tactile stimuli presented to the fingers of the same hand is encoded according to an external reference frame which originates in the centre of the hand (Exp. 3a and $3 b$, but see the general discussion for possible alternative origins of this hand-centered reference frame), this external spatial code is unaffected by the location of the hand with respect to the body midline, as revealed by the results of this experiment (central vs lateral hand position) and of Experiment 1 (crossed vs. uncrossed hands).

\begin{tabular}{|c|c|c|c|c|c|c|c|}
\hline & $\begin{array}{c}\text { Number } \\
\text { of } \\
\text { hands }\end{array}$ & $\begin{array}{c}\text { Number } \\
\text { of stimuli } \\
\text { on } \\
\text { each } \\
\text { hand }\end{array}$ & $\begin{array}{l}\text { Hand } \\
\text { distance } \\
\text { from } \\
\text { midline }\end{array}$ & $\begin{array}{c}\text { Hand } \\
\text { position }\end{array}$ & $\begin{array}{c}\text { Hand } \\
\text { posture }\end{array}$ & $\frac{\begin{array}{c}\text { Trunk- } \\
\frac{\text { centered }}{\text { somatotopic }}\end{array}}{\text { SE }}$ & $\begin{array}{c}\begin{array}{c}\text { Hand- } \\
\text { centered }\end{array} \\
\text { external SE }\end{array}$ \\
\hline \multirow[t]{2}{*}{ Exp. 1} & Two & Two & $30 \mathrm{~cm}$ & Uncrossed & Palm-down & $\begin{array}{l}45 \mathrm{~ms}^{* * *} \\
(4.4 \% * * *)\end{array}$ & $\begin{array}{c}9 \mathrm{~ms} * * \\
(0.2 \%)\end{array}$ \\
\hline & Two & Two & $30 \mathrm{~cm}$ & Crossed & Palm-down & $\begin{array}{c}10 \mathrm{~ms} * \\
(1.3 \%)\end{array}$ & $\begin{array}{c}16 \mathrm{~ms}^{* * *} \\
(2.4 \% * *)\end{array}$ \\
\hline \multirow[t]{2}{*}{ Exp. 2} & One & Two & $25 \mathrm{~cm}$ & Uncrossed & Palm-down & $\begin{array}{c}7 \mathrm{~ms} \\
(2.5 \%)\end{array}$ & $\begin{array}{l}19 \mathrm{~ms} * * \\
(2.7 \% * *)\end{array}$ \\
\hline & Two & Two & $25 \mathrm{~cm}$ & Uncrossed & Palm-down & $\begin{array}{l}31 \mathrm{~ms}^{* * *} \\
(4.8 \% * * *)\end{array}$ & $\begin{array}{l}12 \mathrm{~ms} \\
(1.1 \%)\end{array}$ \\
\hline \multirow[t]{2}{*}{ Exp. 3a } & One & Two & $0 \mathrm{~cm}$ & Uncrossed & Palm-down & $\begin{array}{c}4 \mathrm{~ms} \\
(0.4 \%)\end{array}$ & $\begin{array}{c}45 \mathrm{~ms} * * * \\
(2.5 \% * *)\end{array}$ \\
\hline & One & Two & $0 \mathrm{~cm}$ & Uncrossed & Palm-up & $\begin{array}{l}8 \mathrm{~ms} * \\
(0.6 \%)\end{array}$ & $\begin{array}{l}33 \mathrm{~ms} * * * \\
(2.9 \% * * *)\end{array}$ \\
\hline \multirow[t]{2}{*}{ Exp. 3b } & One & Two & $25 \mathrm{~cm}$ & Uncrossed & Palm-down & $\begin{array}{c}1 \mathrm{~ms} \\
(0.0 \%)\end{array}$ & $\begin{array}{c}37 \mathrm{~ms}^{* * *} \\
(2.9 \% * * *)\end{array}$ \\
\hline & One & Two & $25 \mathrm{~cm}$ & Uncrossed & Palm-up & $\begin{array}{c}5 \mathrm{~ms} \\
(1.2 \%)\end{array}$ & $\begin{array}{c}24 \mathrm{~ms}^{* * *} \\
(2.8 \% * *)\end{array}$ \\
\hline Exp. 4 & One & Two & $0 \mathrm{~cm}$ & Uncrossed & Palm-down & $0 \mathrm{~ms}$ & $27 \mathrm{~ms}^{* * *}$ \\
\hline
\end{tabular}




$$
\begin{array}{|lllll} 
& & & (1.0 \%) \\
\text { One } \quad \text { Two } & & & \\
& & & \mathrm{ms} \\
& \mathrm{cm} \quad \text { Uncrossed } & \text { Palm-down } & (0.0 \%)
\end{array}
$$

$(3.6 \% * * *)$

$22 \mathrm{~ms} * * *$

Table 1: A summary of each experiment with different conditions and the magnitude of the trunkcentered somatotopic Simon effect and hand-centered external Simon effect for reaction time and accuracy. Number of asterisks denotes p-values $(* \mathrm{P}<0.05, * * \mathrm{P}<0.01, * * * \mathrm{P}<0.001)$ from paired t-tests.

\section{General discussion}

In a series of four experiments using the tactile Simon effect, we found evidence for coding stimulus position in multiple frames of reference, and that the intensity and/or existence of the tactile Simon effect can vary under different conditions. In Experiment 1, we placed two tactile stimulators (one on the thumb, and one on the little finger) on each hand, with the hands either crossed or uncrossed. In this experiment, we found both a significant somatotopic trunk-centered effect (with participants faster when the stimulus and response were on the same hand/foot) and a significant hand-centered Simon effect (with participants faster when the stimulus was on the same side of the hand as the response foot). In Experiment 2, we contrasted performance with two hands at once versus with only one hand tested at a time. In both conditions, we found a significant hand-centered Simon effect. Interestingly, however, there was no somatotopic Simon effect when only one hand was tested, demonstrating that the somatotopic Simon effect is not obligatory. In Experiments $3 a$ and 3b, we examined the "one hand" condition in more detail, while also manipulating hand posture (palm up, palm down) to identify how left and right are assigned in the hand-centered Simon effect. As in Experiment 2 with one hand, we found a strong hand-centered Simon effect and mixed evidence for a somatotopic Simon effect (finding a small but significant effect in Experiment 3a, but no significant effect in Experiment 3b). We also found strong evidence that left and right are assigned based on external space. Finally, in Experiment 4, we examined whether varying hand position (central, lateral) influenced the hand-centered Simon effect with only one hand tested. As before, we found a strong hand-centered Simon effect, no trunk-centered Simon effect, and no effect of posture on either. The results for all of the experiments are summarized in Table 1. These results have implications for the design of tactile interfaces that present multiple stimuli to the hand, such that any spatial information communicated to the user should be congruent in a hand-centered frame of reference. 


\subsection{Hand-centered Simon effect with external left-right coding}

First, we found novel evidence for a significant hand-centered Simon effect that was replicated across five different experiments. Furthermore, we also manipulated hand posture (palm up vs. down in Experiments $3 \mathrm{a}$ and $3 \mathrm{~b}$ ), to examine how left and right are encoded within the hand. One possibility is that left-right assignment is anatomically fixed to a specific side of the hand, perhaps based on its typical orientation. For example, the left thumb could be consistently coded as "right", regardless of the hand's orientation. Instead, we found that left and right are assigned in external space, inherited from the participant's own trunk. When the hands are palm down, the left thumb is coded as "right", whereas when they are palms up, it is encoded as "left". These results are generally consistent with findings from individuals with tactile extinction who have demonstrated a deficit in a hand-centered reference frame with left-right encoded based on an external assignment system (e.g. Moscovitch \& Behrmann, 1994).

Interestingly, the hand-centered Simon effect was strongly modulated by hand posture (palm up or down), but relatively unaffected by other postural changes involving the limbs and the position of the hand with respect to the body midline or to the head/eyes (crossed vs uncrossed hands in Expt. 1; hand central vs. hand lateral in Expt. 4) when the hand orientation remained unchanged (palm down). Although this provides evidence that tactile stimulus codes can be generated based on a hand-centered reference frame, we note that these results do not unequivocally provide evidence for a hand-centered representation from which these stimulus codes are generated. For example, in these manipulations, a hand-centered frame of reference can also overlap with two other reference frames. One possibility is that when a tactile stimulus is presented to a hand, the participant immediately attends to the center of the hand. If so, then the stimuli could be encoded in an attention-centered frame of reference. Nicoletti \& Umilta (1989) found evidence for a visual Simon effect that encoded stimulus location based on an attention-centered frame of reference. Therefore, our observed results could be due to stimulus codes being generated based on a tactile or multimodal attention-centered representation. A second possibility is that these stimulus codes are generated based on a representation that encodes the relative position of the two stimuli. For example, a subject with left tactile extinction was presented with bilateral stimuli to both sides of the hand and finger in various positions (Tinazzi et al., 2000). For example, bilateral stimuli were presented to the thumb and pinky while manipulating the stimulated hand (left or right), hand orientation (supinated versus pronated), and position of hand relative to the trunk (left or right). Furthermore, the subject was presented with tactile stimuli to the left and right side of each index finger 
in the pronated and supinated orientations. The subject extinguished the more contralesional stimulus in every manipulation. The authors suggest tactile stimuli can be encoded based on reference frames that dynamically scale from a trunk-centered midline to other body-part centered midlines (hand-centered, finger-centered, etc.). Our study does not differentiate between these reference frames. However, this will be addressed in future work.

\subsection{Multiple stimulus codes and salience}

We found evidence that multiple tactile stimulus codes could be generated for the same stimulus, as we found concurrent somatotopic trunk-centered and hand-centered Simon effects in the experiments in which two tactile stimulators were attached to each hand (Experiments 1 and 2). These results are consistent with findings in the visual Simon effect literature in which multiple stimulus codes were concurrently generated based on hemifield, hemispace, and relative-position based frames of reference (Lamberts et al., 1992; Roswarski \& Proctor, 1996; Rubichi, Nicoletti, \& Umilta, 2005; Umilta \& Liotti, 1987). Our findings provide evidence that automatic stimulus codes can be generated based on both a somatotopic representation and a second, potentially hand-centered representation with external left-right assignment. Previous studies investigating the explicit coding of stimulus location have consistently reported a dissociation between the reference frames responsible for the spatial coding of stimuli to the fingers and to the hand (e.g. De Haan, Anema, \& Dijkerman, 2012; Haggard, Kitadono, Press, \& TaylorClarke, 2006; Heed, Backhaus, \& Röder, 2012; Tamè, Farnè, \& Pavani, 2011). Although the specific reference frames observed were highly dependent on the task requirements, this dissociation suggests the presence of distinct spatial representations of the body encoding different body regions as well as different body sides. The fact that the spatial codes we observed are not only based on different reference points (trunk vs. hand) but also on different spatial coordinates (somatotopic vs. external) supports the idea of multiple spatial representations of the body.

Furthermore, we also found that the somatotopic trunk-centered Simon effect is strongly influenced by experimental context. These results are generally consistent with findings both within (Hommel, 1993) and across modalities (Ruzzoli \& Soto-Faraco, 2017; Castro, 2018) demonstrating that the Simon effect can be modulated by the nature of the task. -In four experiments in which the participant was tested on only one hand during a block, we found that the somatotopic Simon effect was either not significant (Experiments 2, 3b and 4) or significant, but smaller than what's typically observed with two hands (Experiment 3a). Given the large number of participants tested in a one-hand condition and the 
mixed results, we wanted to examine whether there was a somatotopic Simon effect when combining data from all four experiments. In a post-hoc paired t-test, we found a small but significant Simon effect, $\mathrm{t}(103)=3.16, \mathrm{p}=.002$, as participants were $5.0 \mathrm{~ms}$ faster on somatotopically congruent trials $(619.5 \mathrm{~ms})$ compared to somatotopically incongruent trials $(624.5 \mathrm{~ms})$. This suggests that the somatotopic Simon effect still remains even when being tested with one hand.

Past studies using visual stimuli have shown that stimulus codes can be generated based on the receptor surface. Valle-Inclan, Hackley \& de Labra (2003) presented monocular stimuli to participants in a Simon effect task, where they made lateralized responses to different colors. Importantly, the stimuli were presented at fixation, with no manipulation of the perceived spatial location of the stimuli. They found a significant Simon effect based on the stimulated eye, both in participants who could judge which eye was stimulated, and those that could not (see also Valle- Inclán, Sohn, \& Redondo, 2008). In a related study, Schankin, Valle-Inclan \& Hackley (2010) found that this receptor-based Simon effect was independent of the typical spatial Simon effect. We suggest that the somatotopic Simon effect may be from stimulus codes generated using information that encode hand laterality.

However, this $5 \mathrm{~ms}$ somatotopic Simon effect is quite small compared to the somatotopic Simon effect observed in previous studies (which is approximately $45 \mathrm{~ms}$ ). Why would there be such a reduction in the size of the somatotopic Simon effect? Evidence from the visual Simon effect suggests that when multiple spatial codes are generated (for example when visual stimuli are encoded according to both the horizontal, left vs. right, and the vertical axis, top vs. bottom), the codes from one spatial dimension prevails over the codes from the other dimension (see Rubichi, Vu, Nicoletti, \& Proctor, 2006 for a review). That is, potential conflict between different spatial codes is solved by prioritizing one of the two dimensions. Importantly, which dimension will prevail is strongly influenced by the specific spatial parameters of the task, such as the location or distance between stimuli or between responses, the number and location of the effectors, etc. (Ansorge \& Wuhr, 2004; Memelink \& Hommel, 2005; Proctor, Vu, \& Nicoletti, 2003). In the tactile Simon task, when stimuli are presented to the same hand (one-hand Simon task), the participant does not need to attend to the unstimulated hand. However, the spatial location of the two stimuli on one hand, although not task relevant, has a clear impact on participants' performance. We suggest that with one hand tested, the somatotopic stimulus codes are highly diminished due to a lack of spatial salience, resulting in a substantially decreased somatotopic Simon effect. In line with previous studies of the visual Simon effect, manipulations of salience resulted in changes in the size of the Simon 
effect. Thus, the specific task requirements (one or two hands relevant) can modulate the relative strength or salience of different reference frames.

\subsection{Decreased somatotopic Simon effect with the arms crossed}

Finally, we found that the somatotopic Simon effect (in trials with two stimulators on each hand) significantly decreased with the arms crossed $(10 \mathrm{~ms})$ versus when the arms were uncrossed ( $45 \mathrm{~ms}$; see Experiment 1). This contrasts past findings with one stimulator on each hand, where there was no change in the somatotopic Simon effect based on hand posture (e.g. Medina et al., 2014; Ruzzoli \& Soto-Faraco, 2017). Why would manipulating hand posture influence the somatotopic Simon effect with two stimulators on each hand, but not with one stimulator on each hand?

One possibility is that changes in body posture modulate the relative salience of the different spatial representations (dimensions) when multiple codes are active (i.e. two stimulus locations on each hand). Experiment 1 showed a reduction of the trunk-centered Simon effect with crossed as compared to uncrossed hands in both RTs and error rates. This suggests that the weight of this spatial code was reduced with crossed hands. It is worth noting that while the hand position manipulation (crossed vs uncrossed) did not affect the hand-centered Simon effect as revealed by RTs, the analysis of error rates showed a stronger hand-centered Simon effect with crossed than uncrossed hands, suggesting that the weight of the hand-centered spatial code was increased with crossed hands. Taken together this pattern of results suggests that not only task requirements but also postural changes impact the relative salience of the trunk-centered and hand-centered spatial codes. It is possible that when multiple spatial representations/dimensions are available, departures from a 'default' body posture (hands uncrossed, palm down) result in a selective weight (salience) reduction for the spatial representation affected by the postural manipulation (here the trunk-centered one). That is, crossing the hands might result in decreased salience of the trunk-centered dimension because this postural manipulation reduces the reliability of these codes if/when somatotopic spatial information and postural information are merged. 


\section{Acknowledgements}

We would like to thank Tom Claxton, Jennifer Willbond, Abigail Southgate and Alexandria O'Neal for their help with data collection. This material is based upon work supported by the National Science Foundation under grant no. 1632849. Part of the findings described in this manuscript was presented at the International Multisensory Research Forum, Toronto, in June 2018. 


\section{References}

Ansorge, U., \& Wuhr, P. (2004). A response-discrimination account of the Simon effect. Journal of Experimental Psychology-Human Perception and Performance, 30(2), 365-377.

Castro, L., Soto-Faraco, S., Morís Fernández, L., \& Ruzzoli, M. (2018). The breakdown of the Simon effect in cross-modal contexts: EEG evidence. European Journal of Neuroscience, 47(7), 832844.

De Haan, A. M., Anema, H. A., \& Dijkerman, H. C. (2012). Fingers crossed! An investigation of somatotopic representations using spatial directional judgements. PLOS One, 7(9), e45408.

Haggard, P., Kitadono, K., Press, C., \& Taylor-Clarke, M. (2006). The brain's fingers and hands. Exp Brain Res, 172(1), 94-102. doi:10.1007/s00221-005-0311-8

Hasbroucq, T., \& Guiard, Y. (1992). The Effects of Intensity and Irrelevant Location of a Tactile Stimulation in a Choice Reaction-Time-Task. Neuropsychologia, 30(1), 91-94.

Heed, T., Backhaus, J., \& Röder, B. (2012). Integration of hand and finger location in external spatial coordinates for tactile localization. Journal of Experimental Psychology: Human Perception and Performance, 38(2), 386.

Hommel, B. (1993). Inverting the Simon effect by intention - Determinants of direction and extent of effects of irrelevant spatial information. Psychological Research, 55(4), 270-279.

Lakens, D. (2017). Equivalence tests: a practical primer for tests, correlations, and meta-analyses. Social Psychological and Personality Science, 8(4), 355-362.

Lamberts, K., Tavernier, G., \& d'Ydewalle, G. (1992). Effects of multiple reference-points in spatial stimulus-response compatibility. Acta Psychologica, 79, 115-130.

Medina, J., McCloskey, M., Coslett, H. B., \& Rapp, B. (2014). Somatotopic representation of location: evidence from the Simon effect. J Exp Psychol Hum Percept Perform, 40(6), 2131-2142. doi:10.1037/a0037975

Memelink, J., \& Hommel, B. (2005). Attention, instruction, and response representation. European Journal of Cognitive Psychology, 17(5), 674-685.

Moscovitch, M., \& Behrmann, M. (1994). Coding of Spatial Information in the Somatosensory System Evidence from Patients with Neglect Following Parietal Lobe Damage. Journal of Cognitive Neuroscience, 6(2), 151-155.

Nicoletti, R., \& Umilta, C. (1989). Splitting Visual Space with Attention. Journal of Experimental Psychology-Human Perception and Performance, 15(1), 164-169.

Proctor, R. W., Vu, K. P. L., \& Nicoletti, R. (2003). Does right-left prevalence occur for the Simon effect? Perception \& Psychophysics, 65(8), 1318-1329.

Riggio, L., Gawryszewski, L. D., \& Umilta, C. (1986). What is crossed in crossed-hand effects? Acta Psychologica, 62, 89-100.

Roswarski, T. E., \& Proctor, R. W. (1996). Multiple spatial codes and temporal overlap in choice-reaction times. Psychological Research, 59, 196-211.

Roswarski, T. E., \& Proctor, R. W. (2000). Auditory stimulus-response compatibility: Is there a contribution of stimulus-hand correspondence? Psychological Research-Psychologische Forschung, 63(2), 148-158.

Rubichi, S., Nicoletti, R., \& Umilta, C. (2005). Right-left prevalence with task-irrelevant spatial codes. Psychological Research, 69, 167-178.

Rubichi, S., Vu, K.-P. L., Nicoletti, R., \& Proctor, R. W. (2006). Spatial coding in two dimensions. Psychonomic Bulletin \& Review, 13(2), 201-216.

Ruzzoli, M., \& Soto-Faraco, S. (2017). Modality-switching in the Simon task: The clash of reference frames. Journal of Experimental Psychology: General, 146(10), 1478.

Schankin, A., Valle-Inclan, F., \& Hackley, S. A. (2010). Compatibility between stimulated eye, target location and response location. Psychological Research, 74(3), 291-301. 
Simon, J. R., Hinrichs, J. V., \& Craft, J. L. (1970). Auditory S-R compatibility: Reaction time as a function of ear-hand correspondence and ear-response-location correspondence. Journal of Experimental Psychology, 86, 97-102.

Simon, J. R., \& Rudell, A. P. (1967). Auditory SR compatibility: the effect of an irrelevant cue on information processing. Journal of applied psychology, 51(3), 300.

Simon, J. R., \& Small, A. M. (1969). Processing Auditory Information - Interference from an Irrelevant Cue. Journal of applied psychology, 53(5), 433-435.

Tamè, L., Farnè, A., \& Pavani, F. (2011). Spatial coding of touch at the fingers: Insights from double simultaneous stimulation within and between hands. Neuroscience Letters, 487(1), 78-82.

Tinazzi, M., Ferrari, G., Zampini, M., \& Aglioti, S. M. (2000). Neuropsychological evidence that somatic stimuli are spatially coded according to multiple frames of reference in a stroke patient with tactile extinction. Neuroscience Letters, 287(2), 133-136.

Umilta, C., \& Liotti, M. (1987). Egocentric and Relative Spatial Codes in S-R Compatibility. Psychological Research-Psychologische Forschung, 49(2-3), 81-90.

Valle-Inclan, F., Hackley, S. A., \& de Labra, C. (2003). Stimulus-response compatibility between stimulated eye and response location: implications for attentional accounts of the Simon effect. Psychological Research, 67(4), 240-243.

Valle-Inclán, F., Sohn, F., \& Redondo, M. (2008). Spatial compatibility between stimulated eye and response location. Psychophysiology, 45(2), 279-285.

Wallace, R. J. (1971). S-R compatibility and the idea of a response code. Journal of Experimental Psychology, 88(3), 354-360. 